OPEN ACCESS

Edited by:

Yan Liu,

Imperial College London, United Kingdom

Reviewed by: Alexiane Decout

Imperial College London, United Kingdom Barbara Mulloy,

King's College London, United Kingdom

${ }^{*}$ Correspondence: Maria Cristina Roque-Barreira mcrbarre@fmrp.usp.br

${ }^{\ddagger}$ Present address: Relber Aguiar Gonçales, Life and Health Sciences Research Institute (ICVS), School of Medicine, University of Minho, Braga, Portugal

${ }^{\dagger}$ These authors have contributed equally to this work and share first authorship

Specialty section:

This article was submitted to

Structural Biology,

a section of the journal

Frontiers in Molecular Biosciences

Received: 26 April 2021 Accepted: 16 August 2021 Published: 31 August 2021

Citation:

Pitangui NdeS, Fernandes FF Gonçales RA and Roque-Barreira MC (2021) Virulence Vs. Immunomodulation: Roles of the

Paracoccin Chitinase and

Carbohydrate-Binding Sites in Paracoccidioides brasiliensis Infection.

Front. Mol. Biosci. 8:700797.

doi: 10.3389/fmolb.2021.700797

\section{Virulence Vs. Immunomodulation: Roles of the Paracoccin Chitinase and Carbohydrate-Binding Sites in Paracoccidioides brasiliensis Infection}

\author{
Nayla de Souza Pitangui ${ }^{\dagger}$, Fabrício Freitas Fernandes ${ }^{\dagger}$, Relber Aguiar Gonçales ${ }^{\ddagger}$ and \\ Maria Cristina Roque-Barreira *
}

Department of Cell and Molecular Biology and Pathogenic Bioagents, Ribeirão Preto Medical School, University of São Paulo, Ribeirão Preto, São Paulo, Brazil

Paracoccin (PCN) is a bifunctional protein primarily present in the cell wall of Paracoccidioides brasiliensis, a human pathogenic dimorphic fungus. PCN has one chitinase region and four potential lectin sites and acts as both a fungal virulence factor and an immunomodulator of the host response. The PCN activity on fungal virulence, mediated by the chitinase site, was discovered by infecting mice with yeast overexpressing PCN (PCN-ov). PCN-ov are characterized by increased chitin hydrolysis, a narrow cell wall, and augmented resistance to phagocytes' fungicidal activity. Compared to wild-type (wt) yeast, infection with PCN-ov yeast causes a more severe disease, which is attributed to the increased PCN chitinase activity. In turn, immunomodulation of the host response was demonstrated by injecting, subcutaneously, recombinant PCN in mice infected with wt- $P$. brasiliensis. Through its carbohydrate binding site, the injected recombinant PCN interacts with Toll-like receptor 2 (TLR2) and Toll-like receptor 4 (TLR4) N-glycans on macrophages, triggers M1 polarization, and stimulates protective Th1 immunity against the fungus. The PCNtreatment of wt yeast-infected mice results in mild paracoccidioidomycosis. Therefore, PCN paradoxically influences the course of murine paracoccidioidomycosis. The disease is severe when caused by yeast that overexpress endogenous PCN, which exerts a robust local chitinase activity, followed by architectural changes of the cell wall and release of low size chito-oligomers. However, the disease is mild when exogenous PCN is injected, which recognizes $\mathrm{N}$-glycans on systemic macrophages resulting in immunomodulation.

Keywords: fungal chitinases, paracoccin, virulence factor, immunomodulatory agent, chitinase site, lectin site, P. brasiliensis

\section{INTRODUCTION}

Fungal chitinases (EC 3.2.1.14) hydrolyse chitin, a $\beta$-(1,4)-N-acetyl D-glucosamine homopolymer that is a primary constituent of the fungi cell wall (Puccia et al., 2011). Chitin degradation enables the remodeling and constant plasticity required for cell wall architecture of yeast and filamentous fungi (Adrangi and Faramarzi, 2013; Langner and Gohre, 2016). The identification of fungal chitinases is based on their similarities with the 18 families of bacterial 
or plant chitinases, whose classifications are well established in the literature (Takaya et al., 1998). Fungal chitinases have the highest homology with class III plant chitinases (Hayes et al., 1994), which frequently include carbohydrate-binding modules (CBM). In fungi, the reported CBMs are 1) CBM18 has a chitin-binding domain, 2) CBM1 has a cellulose- and chitin-binding domain; and 3) CBM50 has a peptidoglycanand chitin-binding domain, also known as LysM (Seidl, 2008). Chitinases are classified as glycosyl hydrolases (GH) based on the amino acid sequence similarity of the catalytic domains, the chitinases are distributed in families 18,19 , and 20 of GH (Duo-Chan, 2006). Fungal chitinases belong mainly to family 18 and play relevant roles in nutrition, parasitism, morphogenesis, immunity, and autolysis (Karthik et al., 2014).

Genes encoding chitinases have been identified and characterized in yeast, such as Saccharomyces cerevisiae, Candida albicans (Dunkler et al., 2005), and Kluyveromyces lactis. In filamentous fungi, there are chitinases in the genera Trichoderma, Penicillium, Lecanicillium, Neurospora, Mucor, Beauveria, Lycoperdon, Aspergillus, Myrothecium, Conidiobolus, Metharhizium, Stachybotrys, Agaricus, and Ashbya (Duo-Chuan, 2006; Karthik et al., 2014; Dunkler et al., 2008). In dimorphic fungi, chitinase genes have been reported in Coccidioides immitis (Cole and Hung, 2001), Histoplasma capsulatum (Goughenour et al., 2021), and Paracoccidioides brasiliensis (dos Reis Almeida et al., 2010; Santana et al., 2012; Fernandes et al., 2017).

In the last decade, we identified and characterized a $P$. brasiliensis protein, paracoccin (PCN), which has both lectin (Coltri et al., 2006; Ganiko et al., 2007) and chitinase (dos Reis Almeida et al., 2010) properties. When examined from a fungus-host relationship perspective, PCN was revealed to play roles that are somehow paradoxical. Soluble recombinant $\mathrm{PCN}$, administered to $P$. brasiliensis infected hosts, acts as an immunomodulatory agent that favors protection against the fungus (Alegre-Maller et al., 2014; Alegre et al., 2014). In contrast, endogenous PCN, expressed by the infecting fungus itself, acts as a $P$. brasiliensis virulence factor (Fernandes et al., 2017; Gonçales et al., 2021) that aggravates the infection in a murine model. The first $P$. brasiliensis protein explored concerning this functional duality in the host-pathogen relationship was a surface glycoprotein of $43 \mathrm{kDa}$, known as gp43, which acts as a $P$. brasiliensis adhesin that binds laminin and fibronectin (Vicentini et al., 1994). In addition, a 15-amino acid peptide of gp43 (QTLIAIHTLAIRYAN), designated as P10, was shown to protect a P. brasiliensis infected host against the development of paracoccidioidomycosis (PCM). Additionally, P10 was effective in treating a well-established disease (Magalhães et al., 2012).

This article describes the structural organization of the PCN catalytic and putative carbohydrate-binding regions and the paradoxical effects of each type of region, acting as a $P$. brasiliensis virulence factor and an immunomodulatory agent that induces protection against PCM.

\section{PARACOCCIN STRUCTURAL FEATURES}

In a search for protein regions responsible for the PCN activities, we performed amino acid sequence analysis on the annotated PADG_03,347 sequence (Alegre et al., 2014) retrieved from fungi database site (https://fungidb.org/fungidb/) and examined it with the aid of ExPASy software's (http://www.cbs.dtu.dk/services/SignalP/; https://prosite.expasy.org/). We verified that PADG_03,347 encompassed a signal peptide whose cleavage site was in amino acid residues 22 and 23 and a domain homologous to glycoside hydrolase 18 (GH18) family of chitinases (29-218) whose glutamic acid at position 175 was associated with the catalytic site of the chitinase site (Figure 1A). Since PCN activity depends on the recognition of $\mathrm{N}$-glycans linked to Toll-like receptor 2 (TLR2) and Toll-like receptor 4 (TLR4) (Alegre-Maller et al., 2014), we investigated whether the deduced protein primary structure contained potential carbohydrate-binding sites. Amino acid residue sequences of lectins from different organisms, including fungal proteins, already characterized as having carbohydratebinding sites, were collected from databases and used for comparison with the amino acid sequence of PCN. From analysis at Clustal Omega (https://www.ebi.ac.uk/Tools/msa/clustalo/), we identified the regions between amino acids 30-37, 55-62, 98-107 and 120-126 containing aligned nonpolar amino acid residues, which are frequently involved in carbohydrate recognition. Drickamer and Dood (1999) reported this characteristic set of hydrophobic residues in carbohydrate recognition domains (CRDs) of lectins. More recently, polar amino acids were identified as necessary for the protein-carbohydrate interaction, which involves hydrogen bonding with sugar hydroxyl groups, $\mathrm{CH}-\pi$ interactions with aromatic amino acid side chains, and coordination to calcium ions (Hudson et al., 2015; McMahon et al., 2020). The PCN analysis has also revealed the presence of amino acid residues with polar properties that could potentially contribute to the interaction with carbohydrates. A second PCN analysis, using the Prosite software (https://prosite.expasy.org/), determined two chitooligosaccharides binding regions in amino acid residues 98-99 and 123-126. These observations suggest four potential carbohydrate-binding regions on $\mathrm{PCN}$ and one catalytic site.

We also obtained the three-dimensional molecular model of PCN by submitting its amino acid sequence to the Swiss-model software (https://swissmodel.expasy.org/). The structure used as a template was ChiA1 of Aspergillus fumigatus in complex with acetazolamide (PDB 2XTK) with 47.47\% identity. Figure 1B,C show the localization of the carbohydrate-binding regions predicted from the sequence analysis and the active chitinase site. It is worth highlighting the position of the CRDs converged at the same region of the molecule's three-dimensional structure.

\section{RECOMBINANT PARACOCCIN INDUCES PROTECTIVE IMMUNITY AGAINST FUNGAL INFECTION}

Because of their carbohydrate-binding sites, PCN interacts with GlcNAc monomer and homopolymers (Ganiko et al., 


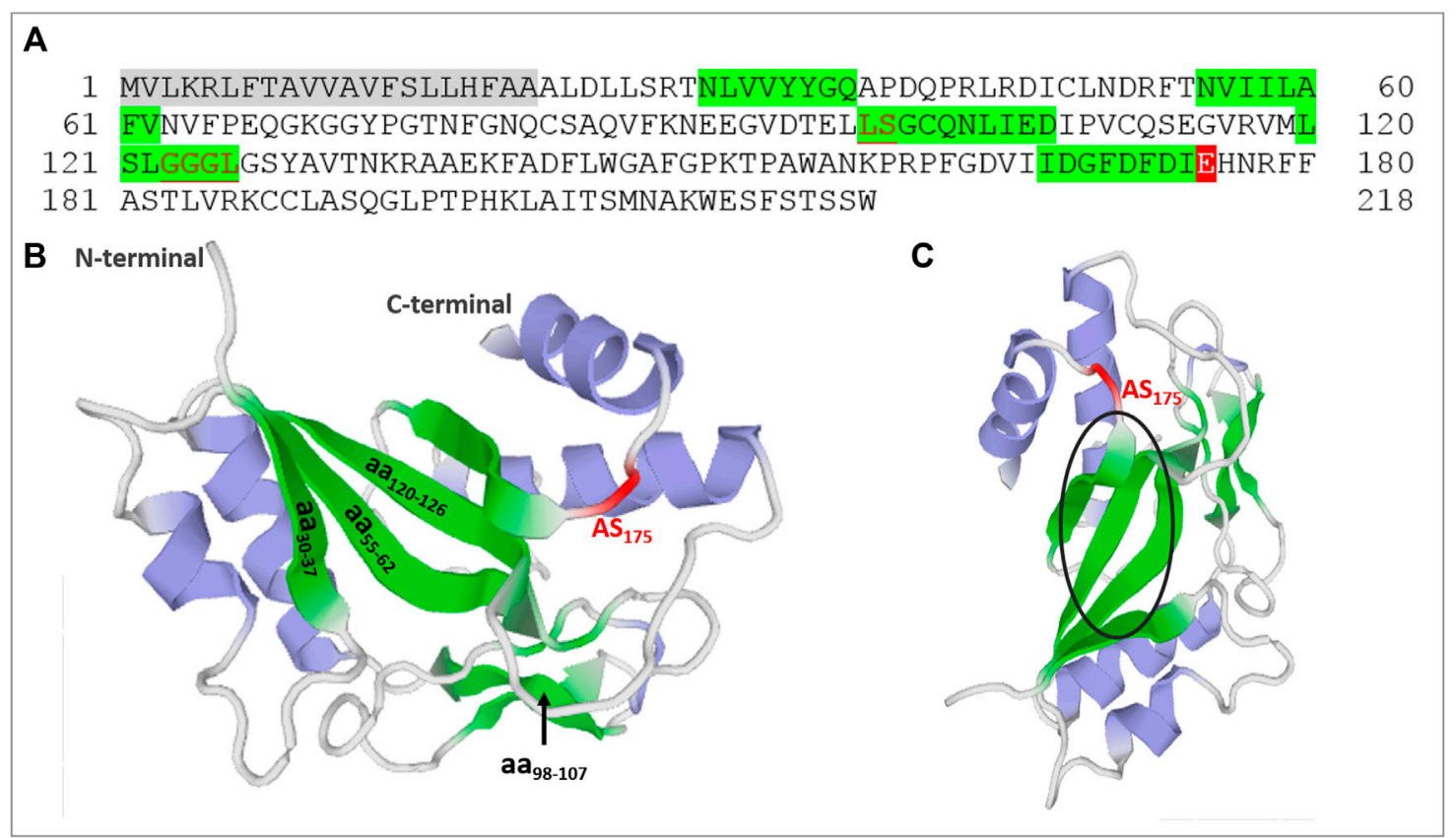

FIGURE 1 | Identification of catalytic and potential carbohydrate-binding regions in paracoccin (PCN). (A) The PCN amino acid residue sequence (PADG_03,347) from $P$. brasiliensis used in protein domain analyzes. The gray is the signal sequence, and the red is the catalytic site of the family 18 chitinases domain. Glutamic acid at position 175 is associated with the catalytic site of the chitinase domain. The green indicates regions of the PCN likely to bind to carbohydrates. The underlined red letters were chito-oligosaccharide-binding regions determined by Prosite software. (B) Molecular model of PCN with potential carbohydrate-binding regions and chitinase active site (AS) identified in green and red. (C) Molecular model of PCN from another angle $\left(90^{\circ}\right)$ with a black circle highlighting the beta sheet tertiary structure in the three-dimensional molecule.

2007; dos Reis Almeida et al., 2010), as well as with GlcNAccontaining $\mathrm{N}$-glycans, such as the ones found in the glycoproteins laminin (Ganiko et al., 2007), TLR-2, and TLR-4 (Alegre-Maller et al., 2014; Freitas et al., 2016). The in vitro demonstration that PCN interaction with TLRs on macrophages induces cell activation and signalling for pro-inflammatory cytokine production, such as IL-12, has encouraged investigations on immunomodulatory property of PCN (Alegre-Maller et al., 2014). Simultaneously, some lectins were isolated from human pathogenic fungi (reviewed by Gallegos et al., 2014). Several reports that lectins from pathogens provide adherence for the pathogen to host epithelial cells suggested that the lectins could account for relevant biological activities and make them attractive targets for developing novel drugs (Houser et al., 2013). Despite this, only a few lectins from pathogenic fungi have been studied in detail until now. In an experimental model of human paracoccidioidomycosis, when recombinant PCN is injected subcutaneously in mice, the course of the disease is milder. Similarly, murine toxoplasmosis is also modified by injecting the recombinant lectins from Toxoplasma gondii, microneme proteins 1 (MIC-1) and 4 (MIC-4) (Lourenço et al., 2006).

PCN administration to $P$. brasiliensis infected mice confers protection against paracoccidioidomycosis through a mechanism primarily triggered by PCN interaction with N-glycans of TLR2 and TLR4 on the surface of macrophages. Detailed studies on TLR2 have identified which $\mathrm{N}$-glycans mediate responses to PCN stimulus. Its effects on transfected cells expressing only a certain TLR2 glycomutant, or a combination of glycomutants, were compared to that exerted-on cells expressing the wt-receptor (Alegre-Maller et al., 2014). We verified that the presence of the fourth $\mathrm{N}$-glycan, among the four TLR2 $\mathrm{N}$-glycans, was enough to induce cell activation. Interestingly, site 4 of TLR2 N-glycosylation is the most conserved and least accessible among all TLR2 N-glycosylation sites (RicciAzevedo et al., 2017). All the TLR2 glycomutants unresponsive to PCN were functional, i.e., mediated cell activation, when stimulated by a classical TLR2 agonist, Pam3CSK4. These findings demonstrated that PCN activity depends on receptor glycosylation. The responsive macrophages responsive to PCN become M1 polarized cells (Freitas et al., 2016) that release cytokines, such as IL-12, which stimulate the development of T helper 1 (Th1) cells (Coltri et al., 2006; Alegre-Maller et al., 2014; Alegre et al., 2014; Freitas et al., 2016). The Th1 cells release IFN- $\gamma$ and are protective against most systemic fungal diseases (Verma et al., 2014). The co-production of high and persistent levels of TNF-a (Coltri et al., 2006) and nitric oxide also contributes to host protection by increasing vascular permeability and tissue accumulation of effector macrophages, with augmented fungicidal activity. Our studies have demonstrated that, by interacting with TLRs N-glycans on macrophages, PCN acts as an immunomodulatory agent to drive the host immune response to a Th1 protective axis. As an efficient immunomodulator, PCN became the focus of further studies designed to elucidate its role in fungal biology and pathogenesis. 


\section{ENDOGENOUS PARACOCCIN FAVORS FUNGUS VIRULENCE AND PATHOGENICITY}

PCN distribution in the cell wall of $P$. brasiliensis hyphae, transition forms, and mature yeast was detected by confocal microscopy using anti-paracoccin antibodies (Ganiko et al., 2007; Oliveira et al., 2017). Fluorescent labelling of PCN occurred predominantly in structures related to fungal growth, such as hyphae tips, chlamydospores of differentiating hyphae, and the budding regions of yeast, supporting the hypothesis that PCN has a function in fungal growth and dimorphic transformation (Ganiko et al., 2007; Oliveira et al., 2017).

To explore the role of $\mathrm{PCN}$ in fungal biology and pathogenesis, we have genetically modified the PCN expression in P. brasiliensis yeast. The difficulties known to be inherent to $P$. brasiliensis molecular manipulation were overcome with the indispensable collaboration of Dr. Fernando Rodrigues (School of Health Sciences, University of Minho, Braga, Portugal) using the technique based on Agrobacterium tumefaciens mediated transformation (ATMT). We successfully modified the gene PADG_03,347 (Gene ID: 22582669) and generated PCN knocked-down (kd-PCN) (Fernandes et al., 2017) and PCN overexpressing (ov-PCN) (Gonçales et al., 2021) yeast. Levels of PCN mRNA expression were reduced $60 \%$ in kd-PCN, and augmented 7-fold in ov-PCN compared to wt-yeast. Consistently, modified PCN protein detection was determined by confocal microscopy and chitinase activity in kd-PCN and ov-PCN yeast.

kd-PCN yeast are very agglomerated, a characteristic attributed to impaired daughter cell detachment due to PCN deficiency. Other authors verified yeast agglomeration in other fungal species where the genes coding for chitinase had been deleted (Kuranda and Robbins, 1991; Dunkler et al., 2005). The decreased PCN expression was also related to P. brasiliensis yeast's incapacity to transition to mycelium. This process primarily depends on coordinated cell wall remodelling chitinase activity (Bastos et al., 2007). In contrast, overexpression of the gene PADG_03,347 allowed a robust separation of the budding yeast from the mother cells. These isolated transformed yeasts displayed reduced cell size and accelerated yeast-to-hypha transition. Increased expression of PCN is readily detectable in the cell wall of ov-PCN yeast, whose chitin content is diminished. Compared to the wt-yeast, the ov-PCN yeast cell wall was about 6 times thinner and was accompanied by a thick outer layer of a fibrillar material, compatible with the presence of a substantial amount of GlcNAc oligomers. Because the ov-PCN total carbohydrate content was higher than that detected in wt-yeasts and PCN has chitinase activity, we examined the particles of $\beta$-1,4-linked GlcNAc oligomers captured by affinity to WGA from the culture supernatants of ov-PCN and wt-yeasts. The particles isolated from wt-PCN varied widely in size and ranged up to $240 \mathrm{~nm}^{2}$, while those from ov-PCN were smaller and had a narrower size distribution ranging up to $60 \mathrm{~nm}^{2}$. PCN overexpression, associated with increased chitinase-activity, leads to greater hydrolysis of the yeast's cell wall chitin into smaller chitooligomers, a reduced $\beta$-1,4-GlcNAc content, and diminished cell wall thickness (Gonçales et al., 2021). These alterations culminate in altering the cell wall architecture.
Functional in vitro assays have shown that PCN-silencing does not affect the adherence or phagocytosis of $P$. brasiliensis yeasts by host cells but renders the yeast more susceptible to the macrophages fungicidal activity (Fernandes et al., 2017). PCNoverexpressing, in turn, augments yeast phagocytosis by macrophages and renders the yeast more resistant to macrophages fungicidal mechanisms (Gonçales et al., 2021).

Mice infected with kd-PCN yeast, compared to wt-yeast, had a mild pulmonary disease, manifested by a low pulmonary fungal burden, few focal inflammatory lesions, and reduced mortality rates (Fernandes et al., 2017). In contrast, mice infected with ov-PCN yeast, compared to wt-yeast, developed a severe systemic disease accompanied by elevated fungal load, extensive and coalescent lung granulomas, and high mortality rates (Gonçales et al., 2021) (Figure 2).

The contrasting disease patterns produced by kd-PCN and ov-PCN yeast are consistent with the verified in vitro features of the transformed yeast, in the sense that kd-PCN yeast are susceptible (Fernandes et al., 2017) and ov-PCN yeasts are resistant to the fungicidal activity of phagocytes (Gonçales et al., 2021).

The host protection conferred by injection of exogenous PCN in P. brasiliensis infected mice was the focus of our initial studies on PCN (Coltri et al., 2006; Alegre et al., 2014; Alegre-Maller et al., 2014; Freitas et al., 2016). These observations lead us to consider PCN as just an immunomodulatory agent that favors host resistance to P. brasiliensis infection (Ricci-Azevedo et al., 2017). Therefore, the results obtained with mice infected with kd-PCN and ov-PCN yeast (Fernandes et al., 2017; Gonçales et al., 2021), seemed to conflict with our assumptions. We expected that ovPCN yeast would cause an auto-resolving illness due to their high content of an agent able to drive host immunity toward a Th1 response. We attributed the mechanism of the protection induced by PCN to the interaction of its CRDs with TLRs $\mathrm{N}$-glycans which leads to phagocyte activation with the subsequent production of TNF- $\alpha$, NO, and IL-12. The culmination of the phagocyte activation is the killing of yeast by macrophages and $\mathrm{T}$ cells. Nonetheless, yeast infection with increased endogenous PCNexpression brought no beneficial effect to the host. On the contrary, as already mentioned, mice infected with ov-PCN yeast became severely ill with a high fungal burden, coalescent pulmonary granulomatous lesions, and a high mortality rate (Gonçales et al., 2021). This disease configuration was further explained by the action of the chitinase domain of endogenous $\mathrm{PCN}$ on the cell wall chitin, increasing the yeast resistance to killing and overcoming the immunomodulatory effect triggered by the eventual binding of PCN lectin sites to host cells' TLRs. The abundant insoluble PCN, localized in the yeast cell wall, does not have easy access to macrophages, whose TLRs $\mathrm{N}$-glycans would be targeted by the PCN lectin domain, as verified for soluble PCN. In the fungal cell wall, PCN intermingles with chitin, a substrate efficiently cleaved by the PCN enzymatic region, allowing the formation of a broad external layer of chito-oligomers. We postulate that the small yeast-derived chito-oligomers, once released, can exert a regulatory role on cytokines production by neighbouring macrophages. 


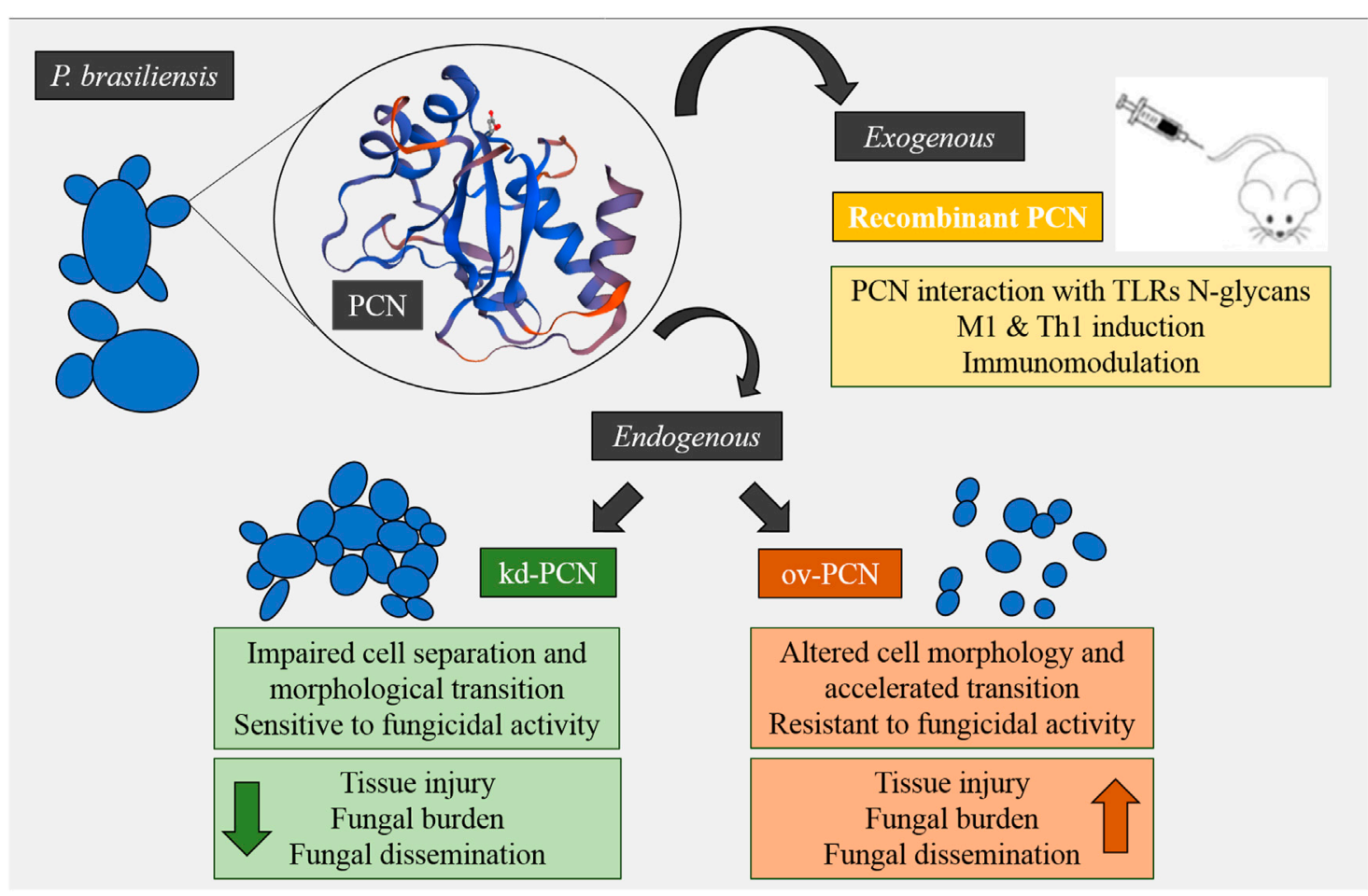

FIGURE 2 | Concise model of the effect of exogenous and endogenous PCN on fungal virulence and modulation of the host's immune response.

Chitin particles are known to induce a wide range of polarized macrophage activation, depending, at least in part, on particle size. Particles larger than macrophages are difficult to phagocytose and are frequently inert in terms of inducing macrophage activation. Differently, microparticles, possessing at least $6 \mathrm{GlcNAc}$ oligomers, are capable of being phagocytosed and activate TLR2 on macrophages and induce their M1 polarization, an event identified by prominent TNF- $\alpha$ production. Smaller chitin particles interact with other pattern recognition receptors and induce M2 macrophages, which produce anti-inflammatory cytokines, such as IL-10. When this occurs in the early phases of the fungal infection, it dampens the Th1 protective immunity response (Costa et al., 2013). Therefore, chito-oligomers can stimulate antagonistic responses depending on their size (Da Silva et al., 2009; Wagener et al., 2014; Fuchs et al., 2018). The severe disease developed by infected mice with ov-PCN yeasts may be due to the already cited resistance to fungicidal mechanisms and IL-10 production, dampening the host's Th1 protective immune response. We expect to answer this question through experiments that are in progress in our laboratory.

Although there are still some mechanistic questions to be answered, previous studies have provided strong evidence that the PCN lectin and enzymatic sites exert opposite effects on the course of $P$. brasiliensis infection.

\section{OUTLOOK}

The observations reviewed herein show that administering the soluble recombinant $P$. brasiliensis component PCN to infected mice benefits the host through actions mediated by the PCN lectin sites. In contrast, augmented expression of endogenous PCN in the infective transformed yeast favors the fungal virulence and pathogenicity through actions mediated by the PCN chitinase site.

\section{AUTHOR CONTRIBUTIONS}

$\mathrm{NP}, \mathrm{FF}$, and MRB conceived the study. NP, FF, and MRB wrote the manuscript. RG contributed to the initial draft of the manuscript and commented on the final manuscript. All authors contributed to the article and approved the submitted version.

\section{FUNDING}

This work was supported by the São Paulo Research Foundation (grants 2017/06251-6, 2018/21708-5 and 2020/16548-9). 


\section{REFERENCES}

Adrangi, S., and Faramarzi, M. A. (2013). From Bacteria to Human: a Journey into the World of Chitinases. Biotechnol. Adv. 31, 1786-1795. doi:10.1016/j.biotechadv.2013.09.012

Alegre, A. C. P., Oliveira, A. F., dos Reis Almeida, F. B., Roque-Barreira, M. C., and Hanna, E. S. (2014). Recombinant Paracoccin Reproduces the Biological Properties of the Native Protein and Induces Protective Th1 Immunity against Paracoccidioides Brasiliensis Infection. Plos Negl. Trop. Dis. 8 (4), e2788. doi:10.1371/ journal.pntd.0002788

Alegre-Maller, A. C. P., Mendonça, F. C., da Silva, T. A., Oliveira, A. F., Freitas, M. S., Hanna, E. S., et al. (2014). Therapeutic Administration of Recombinant Paracoccin Confers protection against Paracoccidioides Brasiliensis Infection: Involvement of TLRs. Plos Negl. Trop. Dis. 8 (12), e3317. doi:10.1371/journal.pntd.0003317

Bastos, K. P., Bailão, A. M., Borges, C. L., Faria, F. P., Felipe, M. S., Silva, M. G., et al. (2007). The Transcriptome Analysis of Early Morphogenesis in Paracoccidioides Brasiliensis Mycelium Reveals Novel and Induced Genes Potentially Associated to the Dimorphic Process. BMC Microbiol. 7, 29. doi:10.1186/1471-2180-7-29

Cole, G. T., and Hung, C.-Y. (2001). The Parasitic Cell wall of Coccidioides Immitis. Med. Mycol. 39, 31-40. doi:10.1080/744118874

Coltri, K. C., Casabona-Fortunato, A. S., Gennari-Cardoso, M. L., Pinzan, C. F., Ruas, L. P., Mariano, V. S., et al. (2006). Paracoccin, a GlcNAc-Binding Lectin from Paracoccidioides Brasiliensis, Binds to Laminin and Induces TNF-a Production by Macrophages. Microbes Infect. 8, 704-713. doi:10.1016/j.micinf.2005.09.008

Costa, T. A., Bazan, S. B., Feriotti, C., Araújo, E. F., Bassi, Ê. J., Loures, F. V., et al. (2013). In Pulmonary Paracoccidioidomycosis IL-10 Deficiency Leads to Increased Immunity and Regressive Infection without Enhancing Tissue Pathology. Plos Negl. Trop. Dis. 7 (10), e2512. doi:10.1371/ journal.pntd.0002512

Da Silva, C. A., Chalouni, C., Williams, A., Hartl, D., Lee, C. G., and Elias, J. A. (2009). Chitin Is a Size-dependent Regulator of Macrophage TNF and IL-10 Production. J. Immunol. 182 (6), 3573-3582. doi:10.4049/ jimmunol.0802113

dos Reis Almeida, F. B., de Oliveira, L. L., de Sousa, M. V., Barreira, M. C. R., and Hanna, E. S. (2010). Paracoccin fromParacoccidioides Brasiliensis; Purification through Affinity with Chitin and Identification ofN-Acetyl- $\beta$-D-Glucosaminidase Activity. Yeast 27, 67-76. doi:10.1002/yea.1731

Drickamer, K., and Dodd, R. B. (1999). C-type Lectin-like Domains in Caenorhabditis elegans: Predictions from the Complete Genome Sequence. Glycobiology 9, 1357-1369. doi:10.1093/glycob/9.12.1357

Dünkler, A., Jorde, S., and Wendland, J. (2008). An Ashbya Gossypii Cts2 Mutant Deficient in a Sporulation-specific Chitinase Can Be Complemented by Candida Albicans CHT4. Microbiol. Res. 163, 701-710. doi:10.1016/j.micres.2008.08.005

Dünkler, A., Walther, A., Specht, C. A., and Wendland, J. (2005). Candida Albicans CHT3 Encodes the Functional Homolog of the Cts1 Chitinase of Saccharomyces cerevisiae. Fungal Genet. Biol. 42, 935-947. doi:10.1016/ j.fgb.2005.08.001

Duo-Chuan, L. (2006). Review of Fungal Chitinases. Mycopathologia 161, 345-360. doi:10.1007/s11046-006-0024-y

Fernandes, F. F., Oliveira, A. F., Landgraf, T. N., Cunha, C., Carvalho, A., Vendruscolo, P. E., et al. (2017). Impact of Paracoccin Gene Silencing on Paracoccidioides Brasiliensis Virulence. MBio 8, 537-517. doi:10.1128/ mBio.00537-17

Freitas, M. S., Oliveira, A. F., da Silva, T. A., Fernandes, F. F., Gonçales, R. A., Almeida, F., et al. (2016). Paracoccin Induces M1 Polarization of Macrophages via Interaction with TLR4. Front. Microbiol. 7, 1003. doi:10.3389/fmicb.2016.01003

Gallegos, B., Martínez, R., Pérez, L., Del Socorro Pina, M., Perez, E., and Hernández, P. (2014). Lectins in Human Pathogenic Fungi. Rev. Iberoam. Micol. 31, 72-75. doi:10.1016/j.riam.2013.09.010
Ganiko, L., Puccia, R., Mariano, V., Santanna, O., Freymüller, E., Roquebarreira, M., et al. (2007). Paracoccin, an N-Acetyl-Glucosamine-Binding Lectin of Paracoccidioides Brasiliensis, Is Involved in Fungal Growth. Microbes Infect. 9, 695-703. doi:10.1016/j.micinf.2007.02.012

Gonçales, R. A., Ricci-Azevedo, R., Vieira, V. C. S., Fernandes, F. F., Thomaz, S. M. d. O., Carvalho, A., et al. (2021). Paracoccin Overexpression in Paracoccidioides Brasiliensis Enhances Fungal Virulence by Remodeling Chitin Properties of the Cell wall. J. Infect. Dis. 224, 164-174. doi:10.1093/infdis/jiaa707

Goughenour, K. D., Whalin, J., Slot, J. C., and Rappleye, C. A. (2021). Diversification of Fungal Chitinases and Their Functional Differentiation in Histoplasma Capsulatum. Mol. Biol. Evol. 38, 1339-1355. doi:10.1093/molbev/msaa293

Hayes, C. K., Klemsdal, S., Lorito, M., Di Pietro, A., Peterbauer, C., Nakas, J. P., et al. (1994). Isolation and Sequence of an Endochitinase-Encoding Gene from a cDNA Library of Trichoderma harzianum. Gene 138, 143-148. doi:10.1016/0378-1119(94)90797-8

Houser, J., Komarek, J., Kostlanova, N., Cioci, G., Varrot, A., Kerr, S. C., et al. (2013). A Soluble Fucose-specific Lectin from Aspergillus fumigatus Conidia - Structure, Specificity and Possible Role in Fungal Pathogenicity. PLoS One 8, e83077. doi:10.1371/ journal.pone.0083077

Hudson, K. L., Bartlett, G. J., Diehl, R. C., Agirre, J., Gallagher, T., Kiessling, L. L., et al. (2015). Carbohydrate-Aromatic Interactions in Proteins. J. Am. Chem. Soc. 137, 15152-15160. doi:10.1021/jacs.5b08424

Karthik, N., Akanksha, K., and Pandey, A. (2014). Production, Purification and Properties of Fungal Chitinases-Aa Review. Indian J. Exp. Biol. 52, 1025-1035.

Kuranda, M. J., and Robbins, P. W. (1991). Chitinase Is Required for Cell Separation during Growth of Saccharomyces cerevisiae. J. Biol. Chem. 266, 19758-19767. doi:10.1016/s0021-9258(18)55057-2

Langner, T., and Göhre, V. (2016). Fungal Chitinases: Function, Regulation, and Potential Roles in Plant/pathogen Interactions. Curr. Genet. 62, 243-254. doi:10.1007/s00294-015-0530-x

Lourenço, E. V., Bernardes, E. S., Silva, N. M., Mineo, J. R., Panunto-Castelo, A., and Roque-Barreira, M.-C. (2006). Immunization with MIC1 and MIC4 Induces Protective Immunity against Toxoplasma Gondii. Microbes Infect. 8, 1244-1251. doi:10.1016/j.micinf.2005.11.013

Magalhães, A., Ferreira, K. S., Almeida, S. R., Nosanchuk, J. D., Travassos, L. R., and Taborda, C. P. (2012). Prophylactic and Therapeutic Vaccination Using Dendritic Cells Primed with Peptide 10 Derived from the 43-kilodalton Glycoprotein of Paracoccidioides Brasiliensis. Clin. Vaccin. Immunol. 19, 23-29. doi:10.1128/CVI.05414-11

McMahon, C. M., Isabella, C. R., Windsor, I. W., Kosma, P., Raines, R. T., and Kiessling, L. L. (2020). Stereoelectronic Effects Impact Glycan Recognition. J. Am. Chem. Soc. 142, 2386-2395. doi:10.1021/jacs.9b11699

Oliveira, A. F., Fernandes, F. F., Mariano, V. S., Almeida, F., Ruas, L. P., Oliveira, L. L., et al. (2017). Paracoccin Distribution Supports its Role in Paracoccidioides Brasiliensis Growth and Dimorphic Transformation. PLoS One 12, e0184010. doi:10.1371/journal.pone.0184010

Puccia, R., Vallejo, M. C., Matsuo, A. L., and Longo, L. V. G. (2011). The Paracoccidioides Cell wall: Past and Present Layers toward Understanding Interaction with the Host. Front. Microbio. 2, 257. doi:10.3389/ fmicb.2011.00257

Ricci-Azevedo, R., Roque-Barreira, M.-C., and Gay, N. J. (2017). Targeting and Recognition of Toll-like Receptors by Plant and Pathogen Lectins. Front. Immunol. 8, 1820. doi:10.3389/fimmu.2017.01820

Santana, L. A. d. P., Vainstein, M. H., Tomazett, P. K., Santos-Silva, L. K., Góes, A. M., Schrank, A., et al. (2012). Distinct Chitinases Are Expressed during Various Growth Phases of the Human Pathogen Paracoccidioides Brasiliensis. Mem. Inst. Oswaldo Cruz 107, 310-316. doi:10.1590/S007402762012000300004

Seidl, V. (2008). Chitinases of Filamentous Fungi: a Large Group of Diverse Proteins with Multiple Physiological Functions. Fungal Biol. Rev. 22, 36-42. doi:10.1016/j.fbr.2008.03.002

Takaya, N., Yamazaki, D., Horiuchi, H., Ohta, A., and Takagi, M. (1998). Cloning and Characterization of a Chitinase-Encoding Gene (chiA) fromAspergillus Nidulans, Disruption of Which Decreases Germination 
Frequency and Hyphal Growth. Biosci. Biotechnol. Biochem. 62, 60-65. doi:10.1271/bbb.62.60

Verma, A., Wüthrich, M., Deepe, G., and Klein, B. (2014). Adaptive Immunity to Fungi. Cold Spring Harbor Perspect. Med. 5, a019612. doi:10.1101/ cshperspect.a019612

Vicentini, A. P., Gesztesi, J. L., Franco, M. F., de Souza, W., de Moraes, J. Z., Travassos, L. R., et al. (1994). Binding of Paracoccidioides Brasiliensis to Laminin through Surface Glycoprotein Gp43 Leads to Enhancement of Fungal Pathogenesis. Infect. Immun. 62 (4), 1465-1469. doi:10.1128/iai.62.4.1465-1469.1994

Conflict of Interest: The authors declare that the research was conducted in the absence of any commercial or financial relationships that could be construed as a potential conflict of interest.
Publisher's Note: All claims expressed in this article are solely those of the authors and do not necessarily represent those of their affiliated organizations, or those of the publisher, the editors and the reviewers. Any product that may be evaluated in this article, or claim that may be made by its manufacturer, is not guaranteed or endorsed by the publisher.

Copyright () 2021 Pitangui, Fernandes, Gonçales and Roque-Barreira. This is an open-access article distributed under the terms of the Creative Commons Attribution License (CC BY). The use, distribution or reproduction in other forums is permitted, provided the original author(s) and the copyright owner(s) are credited and that the original publication in this journal is cited, in accordance with accepted academic practice. No use, distribution or reproduction is permitted which does not comply with these terms. 